\title{
Heat treatment parameters effecting the fractal dimensions of AuGe metallization on GaAs
}

\author{
Imre Mojzes, ${ }^{\text {a) }}$ Csaba Dominkovics, and Gábor Harsányi \\ Department of Electronics Technology, Budapest University of Technology and Economics, Goldmann \\ György tér 3, H-1111 Budapest, Hungary \\ Szilvia Nagy \\ Department of Telecommunications, Jedlik Ányos Institute of Informatics, Electrical and Mechanical \\ Engineering, Széchenyi István University, Egyetem tér 1, H-9026 Győr, Hungary \\ János Pipek \\ Department of Theoretical Physics, Institute of Physics, Budapest University of Technology and Economics, \\ H-1521 Budapest, Hungary \\ László Dobos \\ Research Institute for Technical Physics and Material Sciences, Hungarian Academy of Sciences, P.O. Box \\ 49, H-1525 Budapest, Hungary
}

(Received 29 June 2007; accepted 16 July 2007; published online 16 August 2007) Correlation was detected between the thermal treatment parameters of the AuGe-GaAs system and
surface fractal structure. Structural entropic calculations were used to confirm the results obtained
by fractal calculations. () 2007 American Institute of Physics. [DOI: 10.1063/1.2768911]

Compound semiconductor devices require Ohmic contacts that have improved characteristics. In the course of the development of such devices, different material systems, specifically Au-, Ag-, and Al-based alloys have been analyzed. Analysis of the various material systems found Aubased alloys to be the most reliable. Among the Au-based alloys, the AuGeNi contact system proved to offer the best properties, however; in some cases if $\mathrm{Ni}$ is replaced by another layer, the contact performs rather well, too. Cobalt, platinum, and other thin films ${ }^{1}$ can also be considered. Analytical techniques are important for the characterization of the resulting complex systems, as the metal-compoundsemiconductor contact can consist of as many as four to eight elements. In general, the processes occurring when contact is formed are not equilibrium ones, so phase diagrams of bulk materials and material parameters are generally of no use. According to our results a typical process is the evaporation of the volatile component of the system during heat treatment. ${ }^{2}$ The thin metal film contact that is formed after heat treatment has a characteristic surface structure. It can be described by the toolbox of fractal mathematics, as has been demonstrated by Dávid et $a l^{3}$ and Mojzes et $a l .{ }^{4}$ The description only pertains to the layout and did not go as far as analyzing and modeling to clarify the various technical parameters and the emergence of the surface structure.

The experiments involved $n$-GaAs epitaxial structures with carrier concentration of $10^{15} \mathrm{~cm}^{-3}$. An etchant solution of $\mathrm{NH}_{4}: \mathrm{H}_{2} \mathrm{O}_{2}: \mathrm{H}_{2} \mathrm{O}=1: 4: 20$ was used to etch the wafers immediately before evaporation. Deposition took place onto a preheated substrate heated to $200{ }^{\circ} \mathrm{C}$. In order to create the Ohmic contact, the samples thus prepared were exposed to additional thermal treatment in a furnace placed in the workspace of a scanning electron microscope. The heating rate was $150{ }^{\circ} \mathrm{C} / \mathrm{min}$. During thermal treatment the rate of the evaporated volatile component (i.e., arsenic in the case of GaAs) was measured over the sample. Gray scale pictures

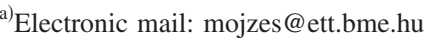

were taken of the surface at regular intervals, and the fractal dimension and the localization properties of the material were analyzed.

Five different algorithms were used to determine the fractal dimensions. One of them, which was developed in our laboratory, used an idea from the wavelet analysis toolbox for giving the fractal dimension, ${ }^{5,6}$ while the other four were modified box counting algorithms. From the two self-made box counting programs one used simplified functions and procedures for the sake of reducing the algorithmical complexity, thus the running time of software decreased. The other two programs were standard FRACLAB routines. First the usability of the programs was tested on a well known object, the so-called Sierpinski triangle, with the dimension of $d=1.5850$.

Only two kinds of image processing methods were applied on the scanning electron microscopy (SEM) pictures before calculating the fractal dimension, decreasing the color depth to $1 \mathrm{bit} / \mathrm{pixel}$ (black and white pictures) or to 8 bit/pixel (gray scale images). The average fractal dimensions produced by the five methods for various heat treating temperatures is shown in Fig. 1. As an example, the standard deviation of the wavelet based method is also given.

For describing the topology free structure of a wave function or any lattice distribution a pair of localization quantities ( $q$ and $S_{\text {str }}$ ) was introduced by Pipek and Varga, ${ }^{7}$ which is calculated for the brightness of the scanning electron microscope pictures of the samples with various heat treatment temperature. The picture forms a lattice with $N$ sites (pixels). The brightness of the pixels $Q_{n}$ is normalized as

$Q_{n} \geqslant 0 \quad$ for $n=1, \ldots, N$,

$\sum_{n=1}^{N} Q_{n}=1$ 


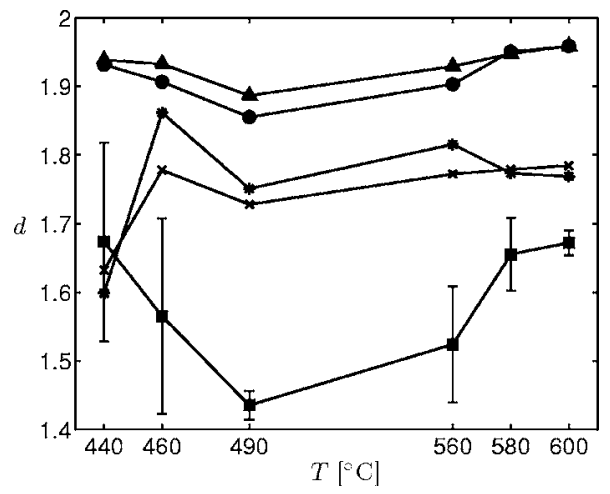

FIG. 1. Correlation between the temperature and the fractal dimension calculated by box counting methods $(*, \boldsymbol{O}, \boldsymbol{\Delta}$, and $\times$ signs $)$ and our wavelet based routine ( $\square$ marker). Error bars are also plotted for the latter data.

The participation ratio or delocalization measure (introduced in Refs. 8 and 9, respectively)

$$
D=\left(\sum_{n=1}^{N} Q_{n}^{2}\right)^{-1}
$$

shows approximately to how many lattice sites the distribution $Q_{n}$ expands. The participation ratio can be normalized by the total number of states $N$, thus the so-called spatial filling factor,

$$
q=\frac{D}{N}
$$

gives the ratio of the bright lattice sites and $q$ fulfills the inequality,

$$
\frac{1}{N} \leqslant q \leqslant 1 .
$$

The spatial filling factor can be used for studying the localization of the distribution $\left\{Q_{n}\right\}$. As the first step, a threshold level $L_{\text {th }}$ was set, the gray scale picture was transformed to black and white, and the filling factor was calculated. Figure 2 shows some typical $q\left(L_{\mathrm{th}}\right)$ diagrams of the heat treated AuGe film on GaAs (100) substrate. The result for both 440 and $600{ }^{\circ} \mathrm{C}$ heat treated samples is a line with more inflexion points, showing that the system is a kind of superstructure. However, for the region between 460 and

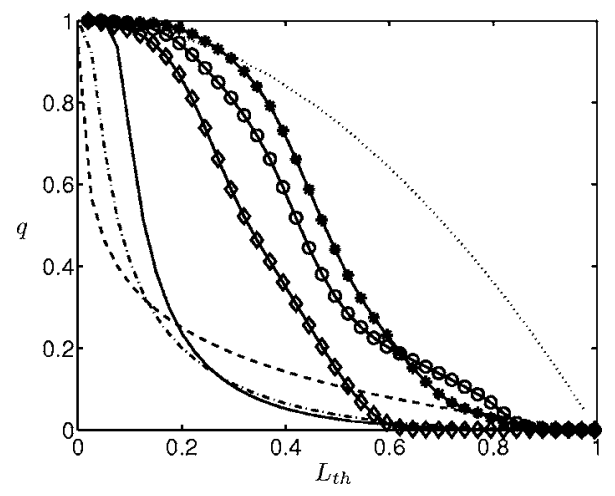

FIG. 2. Filling factors vs the threshold level for AuGe thin film on GaAs substrate at heating temperature of $440{ }^{\circ} \mathrm{C}$ (fractal dimension $d=1.67$ ) plotted with solid line with mark $\diamond$, * and $\bigcirc$ marks mean $490{ }^{\circ} \mathrm{C}(d=1.44)$ and $600{ }^{\circ} \mathrm{C}(d=1.67)$ filling factors, respectively. As a reference the diagrams of a hemisphere (dotted line), a power law function (solid line), an exponential peak (dash-dot line), and a Gaussian function (dashed line) are also shown.

Downloaded 21 Aug 2007 to 152.66.102.96. Redistribution subject

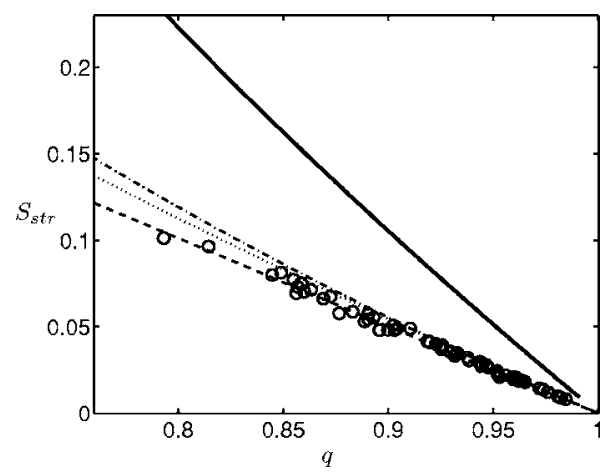

FIG. 3. Structural entropy in terms of the filling factor for the $490{ }^{\circ} \mathrm{C}$ heat treated AuGe layer on GaAs(100) substrate. The limiting line, ln $q$, is plotted with thick solid line. As a reference the localization maps of the Gaussian (dashed line), the exponential (dotted line), and a power law (dash-dot line) localization are also shown.

$580{ }^{\circ} \mathrm{C}$, the filling factor plots have one inflection with characteristics similar to the Gaussian curve. The plateaus at the high and low threshold level values arise from the fact that the pictures have neither absolutely white, nor totally black domains.

The Shannon entropy,

$$
S=-\sum_{n=1}^{N} Q_{n} \ln Q_{n},
$$

characterizes the localization of the lattice distribution: it measures how much the given $\left\{Q_{n} \mid n=1, \ldots, N\right\}$ deviates from the uniform distribution (where all $Q_{n}$ s are the same, $1 / N)$.

The Shannon entropy consists of two parts, the extension and the structural entropies. The extension entropy is derived from the fact that the observed distribution $\left\{Q_{n}\right\}$ is localized to $D$ lattice sites and it is defined as

$$
S_{\text {ext }}=\ln D \text {, }
$$

which is the entropy of a uniform distribution over $D$ sites. The structural entropy corresponds to the shape of the distribution, it characterizes purely the deviation of $\left\{Q_{n}\right\}$ from the step function. The definition of the structural entropy is

$$
S_{\text {str }}=S-\ln D,
$$

and it fulfills the inequality

$$
0 \leqslant S_{\text {str }} \leqslant-\ln q .
$$

The pair ( $q$ and $S_{\text {str }}$ ) is called generalized localization, it describes the topology-free structure of the observed distribution. The distributions $\left\{Q_{n}\right\}$ of a given localization type (e.g., Gaussian) with different filling factors $q$ give a characteristic localization map. The $\left[q\right.$ and $\left.S_{\text {str }}(q)\right]$ values of the SEM pictures of the studied heat treated samples are plotted, and the results were compared with known localization maps; thus the localization type of GaAs films with various treating temperature was given. A typical example of the $S_{\text {str }}(q)$ plots is given in Fig. 3. The dashed, the dotted, and the dash-dot lines in Fig. 3 show the localization maps of the Gaussian, the exponential, and a power law $\left[(1+r)^{-2}\right]$ localization. The dots in the figure follow the Gaussian localization map, as well as the other nondisplayed samples of the temperature between 460 and $560{ }^{\circ} \mathrm{C}$. The pictures of the samples with lower or higher $\left(440\right.$ and $\left.580{ }^{\circ} \mathrm{C}\right)$ temperature
AlP license or copyright, see http://apl.aip.org/apl/copyright.jsp 
thermal treatment are nearer to the exponential map, while the $600{ }^{\circ} \mathrm{C}$ plot is similar to a power law map.

The correlation in Fig. 1 clearly demonstrates that the fractal dimension, i.e., the layout, has a minimum value easy to evaluate. It is to be noted that technologies aimed at optimal contact resistance shows similar minimum values. In this case the optimal temperature necessary to make the emerging metal-compound semiconductor Ohmic contact also shows a characteristic minimum. The AuGe metallization on the compound semiconductor at this temperature leads to optimal specific contact resistance. The localization behavior of the system also changes: in the optimal temperature region the localization is Gaussian while as the deviation from the ideal value increases, the $S_{\text {str }}(q)$ map shows the exponential then the power law characteristics.

In this letter we have studied the fractal and localization properties of heat treated AuGe based Au thin film systems and we have proven that both the value of the fractal dimen- sion and the type of the localization depend on heat treatment temperature.

This work was supported by the Országos Tudományos Kutatási Alap (OTKA) under Grant Nos. T046868 and TO46758.

${ }^{1}$ D. Kumar, Physica A 139, 433 (2006).

${ }^{2}$ I. Mojzes, B. Kovács, M. Schuszter, L. Máté, I. Kun, L. Dobos, and L. Dávid, Thin Solid Films 317, 69 (1998).

${ }^{3}$ L. Dávid, L. Dobos, B. Kovács, I. Mojzes, and B. Pécz, J. Mater. Sci.: Mater. Electron. 17, 321 (2006).

${ }^{4}$ I. Mojzes, S. Kökényesi, I. A. Szabó, I. Iván, and B. Pécz, Nanopages 1, 85 (2006).

${ }^{5}$ F. Argoul, A. Arneodo, J. Elezgaray, and G. Grasseau, Phys. Rev. A 41, 5537 (1990).

${ }^{6}$ J. F. Muzy, E. Bacry, and A. Arneodo, Int. J. Bifurcation Chaos Appl. Sci. Eng. 4, 245 (1994).

${ }^{7}$ J. Pipek and I. Varga, Phys. Rev. A 46, 3148 (1992).

${ }^{8}$ R. J. Bell and P. Dean, Discuss. Faraday Soc. 50, 55 (1970).

${ }^{9}$ J. Pipek, Int. J. Quantum Chem. 36, 487 (1989). 